\title{
Carbapenem stewardship - positive impact on hospital ecology
}

\begin{abstract}
Introduction: Excessive group 2 carbapenem use may result in decreased bacterial susceptibility. Objective: We evaluated the impact of a carbapenem stewardship program, restricting imipenem and meropenem use. Methods: Ertapenem was mandated for ESBL-producing Enterobacteriaceae infections in the absence of non-fermenting Gram-negative bacilli (GNB) from April 2006 to March 2008. Group 2 carbapenems were restricted for use against GNB infections susceptible only to carbapenems and suspected GNB infections in unstable patients. Cumulative susceptibility tests were done for nosocomial pathogens before and after restriction using Clinical and Laboratory Standards Institute (CLSI) guidelines. Vitek System or conventional identification methods were performed and susceptibility testing done by disk diffusion according to CLSI. Antibiotic consumption (t-test) and susceptibilities (McNemar's test) were determined. Results: The defined daily doses (DDD) of group 2 carbapenems declined from 61.1 to 48.7 DDD/1,000 patient-days two years after ertapenem introduction $(p=0.027)$. Mean ertapenem consumption after restriction was $31.5 \mathrm{DDD} / 1,000$ patient-days. Following ertapenem introduction no significant susceptibility changes were noticed among Gram-positive cocci. The most prevalent GNB were P. aeruginosa, Klebsiella pneumoniae, and Acinetobacter spp. There was no change in P. aeruginosa susceptibility to carbapenems. Significantly improved P. aeruginosa and K. pneumoniae ciprofloxacin susceptibilities were observed, perhaps due to decreased group 2 carbapenem use. K. pneumoniae susceptibility to trimethoprim-sulfamethoxazole improved. Conclusion: Preferential use of ertapenem resulted in reduced group 2 carbapenem use, with a positive impact on P. aeruginosa and K. pneumoniae susceptibility.
\end{abstract} Keywords: carbapenems; drug resistance; bacterial ecology.

[Braz J Infect Dis 2011;15(1):1-5] @Elsevier Editora Ltda.

\section{INTRODUCTION}

Susceptibility to the group 2 carbapenems, imipenem and meropenem, remains high even after decades of use. ${ }^{5}$ The more recently introduced group 1 carbapenem ertapenem has a different spectrum of activity, with minimal activity against Pseudomonas aeruginosa; ertapenem has been described as a Pseudomonas-sparing carbapenem. ${ }^{2,12,16}$ The premise that ertapenem has minimal activity against $P$. aeruginosa and is thus less likely to select for resistance has been substantiated in vitro and in clinical settings. ${ }^{4,6,10,11}$ Still, the question of the long-term impact of ertapenem on hospital ecology lingers as overall concerns about antibiotic resistance become more pervasive.

Ertapenem is appropriate for the treatment of extended spectrum beta-lactamase (ESBL)producing Enterobacteriaceae, and thus provides a good weaponry to combat an increasingly important problem, particularly in an era when few new antibiotics are being introduced. The widespread use of ertapenem is likely to depend on long-term clinical evidence of the effect of ertapenem on the susceptibility of Gram-negative bacteria to the spectrum of antibiotics used.

ESBL-producing Enterobacteriaceae are a growing problem in Brazil as they are elsewhere; this problem can result in increased, and not always appropriate, carbapenem use. ${ }^{10,15}$ Antibiotic consumption and the prevalence of antibiotic resistance are linked, giving institutional antibiotic use policies an important role in reducing the selective pressure for resistance., ${ }^{9,15}$ The goal of this study was to evaluate the long-term impact of a carbapenem stewardship program on the hospital ecology at our facility, where ertapenem use was mandated for appropriate infections while group 2 carbapenems were restricted.
Authors

Ana Lucia Lei Munhoz Lima ${ }^{1}$ Priscila Rosalba Domingos de Oliveira $^{2}$

Adriana Pereira de Paula ${ }^{3}$

Karine Dal-Paz

João Nóbrega de Almeida Jr ${ }^{5}$

Cássia da Silva Félix ${ }^{6}$

Flávia Rossi ${ }^{7}$

${ }^{1} \mathrm{MD}, \mathrm{PhD}$ - Chief Physician, Infection Control Service, Institute of Orthopedics and Traumatology Hospital das Clínicas, School of Medicine, Universidade de São Paulo - USP, Brazil

${ }^{2} \mathrm{MD}$ - Assistant Physician, Infection Control Service, Microbiology Laboratory, LIM

${ }^{3} \mathrm{RN}$ - Nurse, Infection Control Service, Hospital das Clínicas, School of Medicine, USP, Brazil ${ }^{4}$ Pharmacist; Infection Service, Institute of Orthopedics and

Traumatology, School of Medicine, USP, Brazil

${ }^{5} \mathrm{MD}$; Assistant Physician,

Microbiology Laboratory, Hospital das Clínicas, Divisão de Laboratório

Central, LIM 03, School of

Medicine, USP, Brazil

${ }^{6} \mathrm{RN}$; Nurse, Infection Service,

Institute of Orthopedics and

Traumatology, Hospital das

Clínicas, School of Medicine,

USP, Brazil

${ }^{7} \mathrm{MD}, \mathrm{PhD}$; Chief Physician,

Microbiology Laboratory, Hospital

das Clínicas, Divisão de Laboratório

Central, LIM 03, School of

Medicine, USP, Brazil

Submitted on: 5/18/2010 Approved on: 8/17/2010

Correspondence to:

Dr. ALLM Lima

Avenida Dr. Ovídio Pires de

Campos, 333 - sala $311^{\mathrm{a}}$. $05403-$

010 - São Paulo

Phone: (55 11) 30696900

Fax: (55 11) 30696900

ccih.iot@hcnet.usp.br

Financial Support: ALLM Lima and $\mathrm{F}$ Rossi are members of a local Merck Sharp \& Dohme

advisory board and have received honoraria for participating in meetings. PR Oliveira, AP Paula, K Dal Paz, JN Almeida Jr and

CS. Felix declare no potential conflicts of interest. The authors wish to thank Wendy Horn,

$\mathrm{PhD}$ of Insight Communication for editorial and writing assistance and Robert Balshaw, $\mathrm{PhD}$ of Syreon Corporation for analytical assistance, which were funded by Merck, Sharp \& Dohme. 


\section{MATERIALS AND METHODS}

\section{Study design}

This single-center study was conducted at the Institute of Orthopedics and Traumatology of Hospital das Clínicas, School of Medicine, Universidade de São Paulo, a 200-bed tertiary care facility that treats orthopedic and trauma patients. A mandatory antibiotic restriction policy was put in place in March 2006. Ertapenem use was mandated for infections caused by ESBL-producing Enterobacteriaceae for patients who did not have co-infection with non-fermenting Gram-negative bacilli. Before the restriction, group 2 carbapenems were used to treat infections caused by ESBL-producing Enterobacteriaceae and infections caused by non-fermenting Gram-negative bacilli that were shown to be susceptible only to carbapenems. Following the restriction group 2 carbapenems were used only to treat infections caused by non-fermenting Gram-negative bacilli infections sensitive to carbapenems alone and to treat suspected Gramnegative infections in hemodynamically unstable patients who did not respond to other agents. Meropenem was not used from March 2005 to March 2006, but was used in the other periods of time included in the present study.

Antimicrobial susceptibility tests were obtained for nosocomial pathogens (e.g. Enterobacteriaceae, Acinetobacter spp., and $P$. aeruginosa) isolated as the cause of nosocomial infection, according to the definitions of the Centers for Disease Control and Prevention (CDC) ${ }^{3}$, for the 24 months before and the 24 months after ertapenem introduction in March 2006. Cumulative susceptibility tests were done for nosocomial pathogens before and after restriction using Clinical and Laboratory Standards Institute (CLSI) guidelines. Strains were identified by Vitek 1 automated microbial identification system. Enterobacteriaceae susceptibility tests were done using the GNS 655 card. Susceptibility to ertapenem was extrapolated from imipenem results, according to CLSI standards during the time this study was performed. ${ }^{13}$ Susceptibility tests for non-fermenting bacteria were done by disk diffusion (CLSI M100-S16).Quality control followed the CLSI standards.

\section{Data analysis}

Antimicrobial susceptibility was measured as the proportion of susceptible isolates of each bacterium to the antimicrobials tested. The statistical significance susceptibility changes before and after the restriction was assessed using the Chisquare test. Consumption of antimicrobial agents was measured as the number of defined daily doses (DDD) per 1,000 patient-days, calculated for each month before and after the restriction. The mean consumptions before and after the restriction were compared using t-tests on the log-transformed data. Generally concordant results were obtained using a t-test performed on the raw data.
Stability of the number of patient-days, length of stay, the numbers of deaths, and the hospital mortality index were also evaluated using these methods. The Durbin-Watson test for autocorrelation was used to rule out the possibility of false positive results that might have been caused by autocorrelation of the monthly data series. The $\mathrm{p}$-values $<0.05$ were considered to be indicative of statistically significant comparisons; these tests are observational, however, and should be interpreted in conjunction with clinical judgment.

\section{RESULTS}

The average defined daily doses (DDD) of group 2 carbapenems declined from 61.1 to $48.7 \mathrm{DDD} / 1,000$ patient-days two years after ertapenem was mandated for treatment of susceptible infections caused by ESBL-producing Enterobacteriaceae $(\mathrm{p}=0.027)$.This represented a $20.2 \%$ decrease in group 2 carbapenem average consumption from the 24 months before the restriction to the 24 months after. Imipenem use decreased by $58.7 \%$; meropenem was used only sporadically during the 24 months prior to the restriction thus the mean change in use was not assessed. The mean ertapenem consumption after the restriction was $31.5 \mathrm{DDD} / 1,000$ patient-days.

No significant antibiotic susceptibility changes were noticed among Gram-positive cocci following ertapenem introduction (Table 1). The most prevalent Gram-negative bacteria that caused infection were P. aeruginosa, Klebsiella pneumoniae, and Acinetobacter spp. There were no changes in $P$. aeruginosa susceptibility to imipenem before and after the restriction (55\% and 57\%, respectively). Improved P. aeruginosa (from $28 \%$ to $57 \%$ ) and K. pneumoniae (from $12 \%$ to $40 \%$ ) ciprofloxacin susceptibilities were observed before and after the restriction, although ciprofloxacin use did not change significantly (before: $41.7 \pm 17.4$ DDD, after: $70.7 \pm 34.5$ DDD; p = 0.057). Although K. pneumoniae susceptibility to trimethoprim-sulfamethoxazole improved from $24 \%$ before the restriction to $60 \%$, trimethoprimsulfamethoxazole consumption was low before and after the restriction $(0.92 \pm 2.27 \mathrm{DDD}$ before to $2.35 \pm 2.97 \mathrm{DDD}$ after, $\mathrm{p}=0.001$ ), so the increase may not be clinically relevant. Acinetobacter spp. susceptibility to carbapenems decreased, a trend also seen in the other departments within our hospital complex that did not have an ertapenem policy use (F Rossi and J N Almeida Jr, unpublished data).

In the 24 months before ertapenem introduction, $45 \%$ of all $P$. aeruginosa isolates were imipenem resistant. In the 24 months after restriction, $43 \%$ of isolates were resistant to imipenem, suggesting that ertapenem did not impact P. aeruginosa susceptibility to imipenem.

The mortality index slightly lower after the restriction was observed (mean \pm SD $0.95 \pm 0.39$ before and $0.71 \pm 0.45$ after the restriction, $\mathrm{p}=0.035$ ). There were no clinically relevant changes in other outcomes. The median length of stay 
Table 1. Antimicrobial susceptibilities for the most frequent agents related to nosocomial infections before and after ertapenem introduction

\begin{tabular}{|c|c|c|}
\hline $\begin{array}{l}\text { Pathogen/Antibiotic } \\
\text { ( } \mathrm{n}^{\circ} \text { isolates collected before/ } \\
\text { after ertapenem policy) }\end{array}$ & $\begin{array}{l}\text { Susceptibility (\%) } \\
2 \text { years before } \\
\text { ertapenem use }\end{array}$ & $\begin{array}{l}\text { Susceptibility (\%) } \\
2 \text { years after } \\
\text { ertapenem use }\end{array}$ \\
\hline \multicolumn{3}{|l|}{ S. aureus $(100 / 76)$} \\
\hline Cephalotin & 30 & 32 \\
\hline Clindamycin & 33 & 32 \\
\hline Methicillin & 30 & 31 \\
\hline Trimethoprim-sulfamethoxazole & 33 & 3 \\
\hline Vancomycin & 100 & 100 \\
\hline \multicolumn{3}{|l|}{ A. baumanii $(64 / 36)$} \\
\hline Amikacin & 15 & 12 \\
\hline Cefepime & 6 & 11 \\
\hline Ceftazidime & 78 & 8 \\
\hline Ceftriaxone & 14 & 3 \\
\hline Ciprofloxacin & 3 & 11 \\
\hline Imipenem & 64 & 33 \\
\hline Trimethoprim-sulfamethoxazole & 6 & 17 \\
\hline \multicolumn{3}{|l|}{ P. aeruginosa $(51 / 42)$} \\
\hline Amikacin & 68 & 67 \\
\hline Cefepime & 43 & 57 \\
\hline Ceftazidime & 61 & 62 \\
\hline Ciprofloxacin & 28 & 57 \\
\hline Imipenem & 55 & 57 \\
\hline \multicolumn{3}{|c|}{ Coagulase-negative Staphylococci (49/39) } \\
\hline Cephalotin & 8 & 8 \\
\hline Clindamycin & 20 & 20 \\
\hline Methicillin & 8 & 8 \\
\hline Trimethoprim-sulfamethoxazole & 43 & 8 \\
\hline Vancomycin & 100 & 100 \\
\hline \multicolumn{3}{|l|}{ E. faecalis $(39 / 28)$} \\
\hline Ampicillin & 92 & 46 \\
\hline Gentamicin & 61 & 25 \\
\hline Penicillin & 4 & 86 \\
\hline Vancomycin & 73 & 79 \\
\hline \multicolumn{3}{|l|}{ K. pneumoniae (25/35) } \\
\hline Amikacin & 76 & 97 \\
\hline Cefepime & 16 & 17 \\
\hline Ceftazidime & 20 & 11 \\
\hline Cefotaxime & 8 & 11 \\
\hline Ceftriaxone & 8 & 9 \\
\hline Ciprofloxacin & 12 & 40 \\
\hline Imipenem & 100 & 100 \\
\hline Trimethoprim-sulfamethoxazole & 24 & 60 \\
\hline
\end{tabular}


was $7.11 \pm 0.74$ days before and $6.89 \pm 0.5$ days after the restriction $(\mathrm{p}=0.306)$; the number of deaths due to infection was $4.71 \pm 2.05$ before and $3.63 \pm 2.41$ after the restriction $(\mathrm{p}=0.074)$. Overall, these data suggest that the severity of illness would have been similar during the two periods and that any observed decrease in Pseudomonas-resistant isolates would not be due to differences in the severity of illness.

\section{DISCUSSION}

Instituting ertapenem use for ESBL-producing Enterobacteriaceae infections in the absence of non-fermenting Gram-negative bacilli while restricting group 2 carbapenems had a positive effect on the overall hospital ecology at our institution. In particular, increased ertapenem use had, in an indirect way, positive impact on the susceptibility of $P$. aeruginosa to imipenem, perhaps related to decreased use of group 2 carbapenems. There was no evidence of carbapenem resistance development associated with ertapenem use. Ertapenem use heralded improvements in the susceptibilities of $P$. aeruginosa and K. pneumoniae to ciprofloxacin, again perhaps due to a decrease in group 2 carbapenem use. An improvement in K. pneumoniae susceptibility to trimethoprim-sulfamethoxazole was noticed. We observed a decrease in Acinetobacter susceptibility to imipenem. In the broader context of data collected from across our institution and analyzed by the Microbiology Laboratory of Hospital das Clinicas (F Rossi and J N Almeida Jr, unpublished data), a downward trend was also observed for susceptibility of Acinetobacter to group 2 carbapenems from 2005 to 2008 despite there being no ertapenem use policy in place. This could suggest that the Acinetobacter susceptibility trends were independent of ertapenem use.

Significant correlations between the consumption of specific antibiotics and resistance have been reported for Gram-negative bacteria including $P$. aeruginosa and Acinetobacter. ${ }^{8,9,13}$ The rate of consumption of specific antibiotics is also related to the prevalence of resistance among nosocomial pathogens. ${ }^{9}$ Examining correlations between consumption of specific antimicrobials and antibiotic resistance can assist in putting effective antimicrobial use policies in place in hospitals. Imipenem resistance, for example, has been linked to the use of amikacin, ciprofloxacin, and ceftazidime, but not to ertapenem. ${ }^{9,11}$ Implementation of interventions such as the one we describe here can have a positive impact on antimicrobial resistance, because inappropriate antibiotic use is a main driver of resistance. ${ }^{15}$ Goldstein et al. reported that adding ertapenem to the formulary in a 344-bed community hospital was an effective antimicrobial management tool. ${ }^{7}$

Apisarnthanarak et al. demonstrated that conducting surveillance and implementing prescribing policies resulted in reductions in antibiotic consumption and resistance in a tertiary care hospital in a developing country. ${ }^{1}$ This is consist- ent with our experience. Our research was part of an ongoing close monitoring of antimicrobial resistance patterns in our hospital following ertapenem introduction. The data reported here were consistent with prior results detailing the impact of ertapenem on hospital ecology within our institution. ${ }^{10,11}$

The policies for rational use of carbapenems should always be encouraged, since it has been observed a worldwide dissemination of multidrug-resistant Gram-negative bacilli, with few therapeutic options. Indiscriminate use of carbapenems is one reason for this problem. It is also necessary to remember the rapidly increasing prevalence of Enterobacteriaceae harboring carbapenemases. From 2000 to 2007, the proportion of carbapenem-resistant Klebsiella spp. involved in nosocomial infections in the United States grew from less than $1 \%$ to $8 \%$ of the total Klebsiella spp. isolates. ${ }^{17}$

Our study provides long-term clinical data indicating that ertapenem is associated with a minimal risk of resistance, and that ertapenem use may improve overall hospital ecology by decreasing excess use of group 2 carbapenems. These results suggest that ertapenem may have an important place in the treatment of ESBL-producing Enterobacteriaceae infections in the absence of Pseudomonas, and that antibiotic use policies that promote stewardship may decrease antibiotic resistance.

\section{ACKNOWLEDGMENTS}

ALLM Lima and F Rossi are members of a local Merck Sharp \& Dohme advisory board and have received honoraria for participating in meetings. PR Oliveira, AP Paula, K Dal Paz, JN Almeida Jr and CS Felix declare no potential conflicts of interest. The authors wish to thank Wendy Horn, PhD of Insight Communication for editorial and writing assistance and Robert Balshaw, PhD of SyreonCorporation for analytical assistance, which were funded by Merck, Sharp \& Dohme.

\section{REFERENCES}

1. Apisarnthanarak A, Danchaivijitr S, Khawcharoenporn T et al. Effectiveness of education and an antibiotic-control program in a tertiary care hospital in Thailand. Clin Infect Dis. 2006; 42:768-775.

2. Brink A, Feldman C, Grolman DC et al. Appropriate use of the carbapenems. S Afr Med J. 2004; 94:857-861.

3. Clinical and Laboratory Standards Institute. 2006. Performance standards for antimicrobial susceptibility testing. Wayne (PA): The Institute; 2006. M100-S16.

4. DiNubile M, Friedland I, Chan CY et al. Bowel colonization with resistant gram-negative bacilli after antimicrobial therapy of intra-abdominal infections: observations from two randomized comparative clinical trials of ertapenem therapy. Eur J Clin Microbiol Infect Dis. 2005; 24:443-449.

5. European Antimicrobial Resistance Surveillance System (EARRS). http://www.rivm.nl/earss/result/Monitoring_reports/Annual_reports.jsp.Accessed 8 July 2009. 
6. Goff DA, Mangino J. Ertapenem: No effect on gram-negative susceptibilities to imipenem. J Infect. 2008; 57:123-127.

7. Goldstein EJ, Citron DM, Peraino V, Elgourt T, Miebohm AR, Lu S. Introduction of ertapenem onto a hospital formulary: Effect on antimicrobial usage improved in vitro susceptibility of Pseudomonas aeruginosa. Antimicrob Agents Chemother. 2009; 53:5122-6.

8. Hsueh PR, Chen WH, Luh KT. Relationships between antimicrobial use and antimicrobial resistance in Gram-negative bacteria causing nosocomial infections from 1991-2003 at a university hospital in Taiwan. Int J Antimicrob Agents 2005; 26:463-472.

9. Iosifidis E, Antachopoulos C, Tsivitanidou M et al. Differential correlation between rates of antimicrobial drug consumption and prevalence of antimicrobial resistance in a tertiary care hospital in Greece. Infect Control Hosp Epidemiol. 2008; 29:615-622.

10. Lima AL, Oliveira PR, Paula AP, and Zumiotti AV. Influence of ertapenem administration on the incidence of carbapenem-resistant Pseudomonas aeruginosa. Braz J Infect Dis. 2008; 12:105-106.

11. Lima AL, Oliveira PR, Paula AP, Dal-Paz K, Rossi F, and Zumiotti AV. The impact of ertapenem use on the susceptibility of Pseudomonas aeruginosa to imipenem: a hospital case study. Infect Control Hosp Epidemiol. 2009; 30:487-90.
12. Livermore D, Mushtaq S, Warner M. Selectivity of ertapenem for Pseudomonas aeruginosa mutants cross-resistant to other carbapenems. J Antimicrob Chemother. 2005; 55:306-311.

13. Loeffler JM, Garbino J, Lew D, Harbarth S, Rohner P. Antibiotic consumption, bacterial resistance and their correlation in a Swiss University Hospital and its Adult Intensive Care Units. Scand J Infect Dis. 2003; 35:843-850.

14. Marra AR, Wey SB, Castelo A et al. Nosocomial bloodstream infections caused by Klebsiella pneumoniae: impact of extended-spectrum $\beta$-lactamase (ESBL) production on clinical outcome in a hospital with high ESBL prevalence. BMC Infect Dis.2006; 6:24-31.

15. Nouwen JL. Controlling antibiotic use and resistance. Clin Infect Dis. 2006; 42:776-777.

16. Shah P, Isaacs RD. Ertapenem, the first of a new group of carbapenems. J Antimicrob Chemother. 2003; 52:538-542.

17. Hirsch E, Tam VH. Detection and treatment options for Klebsiella pneumoniae carbapenemases (KPCs): an emerging cause of multidrug-resistant infection. J Antimicrob Chemother. 2010; 65:1119-25. 